Journal of Mathematics and Statistics 8 (1): 57-63, 2012

ISSN 1549-3644

(C) 2012 Science Publications

\title{
A Study on the Effects of Non-Normality on the Performances of Max-DEWMA Versus SS-DEWMA Charts
}

\author{
Teh Sin Yin and Michael Khoo Boon Chong \\ School of Mathematical Sciences, University Sains Malaysia, \\ 11800 Minden, Penang, Malaysia
}

\begin{abstract}
Problem statement: The called the Sum of Squares Double Exponentially Weighted Moving Average (SS-DEWMA) chart which is effective in detecting shifts in the mean and/or variance is compared with the Max-DEWMA (called the maximum double exponentially weighted moving average) chart. The comparison is based on the assumption that the distribution of the quality characteristic of the process is normal or approximately normally distributed. In many real world situations, this assumption may be violated. This study compares the effects of various forms of nonnormality on the Max-DEWMA and SS-DEWMA control charts. Approach: A Monte Carlo simulation using the Statistical Analysis Software (SAS) is conducted to compare the performances of the two charts for the case of skewed distributions, such as the Weibull, lognormal and gamma distributions. Results: The overall results show that the Max-DEWMA chart has in-control Average Run Lengths (ARLs) closer to the specified value, as compared to that of the SS-DEWMA chart, for all levels of skewnesses considered. Conclusion/Recommendation: Practitioners are advised to use the Max-DEWMA chart for a joint monitoring of the process mean and/or variance, when the underlying distribution is non-normal.
\end{abstract}

Key words: Average Run Length (ARL), DEWMA chart, non-normal distributions, single control chart, industrial processes, standard deviation, control charts, assignable cause, control limits, versus SS-DEWMA charts, statistical control, standard deviation, skewed distributions

\section{INTRODUCTION}

An effective method of process monitoring is by means of using control charts (Oakland, 2003). A control chart is the most powerful tool in Statistical Process Control (SPC) that is extensively used by practitioners to control a variety of industrial processes. The Exponentially Weighted Moving Average (EWMA) chart, introduced by Roberts (1959), for instance, is often used to monitor the location and spread of a process. The EWMA-type control charts are formulated based on the properties of the normal distribution (Duncan, 1986). The normal distribution is described by its parameters, mean $(\mu)$ and standard deviation $(\sigma)$. Since 1930, information gathered from most industries indicates that control limits, based on the 3 standard deviations width provide an economical balance between the costs resulting from the Type I and Type II errors (Umble and Umble, 2000). Unless there are strong practical reasons for using control limits with wider or narrower widths, otherwise the $3 \sigma$ limits should be applied for a normal distribution. As such, 99.73\% of the population points will fall in the interval $(\mu \pm 3 \sigma)$ i.e. within the lower and upper control limits. This means that almost all the population values will fall within the $(\mu \pm 3 \sigma)$ limits if the process is free from any assignable cause, i.e. when no action is needed. In other words, the false alarm rate or size of the Type I error is as low as 1 in every $370(0.27 \%)$ random samples (Besterfield, 2009; Gupta and Walker, 2007).

Recently, the single Max-DEWMA (called the Maximum Double Exponentially Weighted Moving Average) chart proposed by Khoo et al. (2010), which simultaneously detects shifts in the process mean and/or variability was shown to outperform the Max-EWMA chart, proposed by Chen et al. (2001), for small and moderate shifts. More recently, Teh et al. (2010a) proposed the single SS-DEWMA (called the Sum of Squares Double Exponentially Weighted Moving Average) chart which outperforms the SS-EWMA chart proposed by Xie, (1999), in detecting shifts of all sizes in the mean and/or variance. Teh et al. (2010b) conducted a comparative study of the performances of the SS-DEWMA and the Max-DEWMA charts, for monitoring the process mean and/or variance of a normally distributed process. Under the normality assumption, their findings indicated that the SSDEWMA chart is superior to the Max-DEWMA chart, in terms of the out-of-control detection speed and diagnostic abilities.

Corresponding Author: Teh Sin Yin, School of Mathematical Sciences, University Sains Malaysia, 11800 Minden, Penang, Malaysia 
A fundamental assumption that underlies the designs of the Max-DEWMA and SS-DEWMA charts is that the quality characteristic is normally distributed. However, in many situations, the validity of this assumption is doubted, not easy to justify and is often inappropriate. In many industrial processes, the normality assumption of the underlying distribution does not hold. Montgomery (2008) discussed difficulties in the application of statistical control charting techniques to some real data in industries. For example, for a semiconductor laser, the lifetime of a product that degrades over time is often modeled by a lognormal random variable. In reliability engineering, the lifetime and failure rate data for electrical and mechanical components and system follow a Weibull distribution. The failure rate of a product is divided into three phases. The failure rate is high at the beginning (early failure) and at the end (wear out period) of the product cycle. Random or spontaneous failures occur in between the product cycle and this forms a bathtub curve for the failure rate function. Therefore, the Weibull distribution has been used in situations involving electronic devices, such as memory elements; mechanical components like bearings; and structural elements in aircrafts and automobiles.

Hai-Yu and Ji-Chao, (2007) noted that the consequence of applying EWMA charts when the underlying population is skewed is that the Type-I error or false alarm rate (the probability of signaling an outof-control when the process is actually in-control) will increase as the skewness level increases. This is due to the inconsistency between the variability pattern of an asymmetric distribution and the normality assumption in constructing control charts. When this happens, unnecessary process adjustments and loss of confidence in the use of control charts as a monitoring tool will arise. Many studies have recently been made on control charts for skewed populations (Amhemad, 2009; Khoo and Kassim, 2008; Hai-Yu and Ji-Chao, (2007).

In this study, the effect of departures from normality on both the Max-DEWMA and SS-DEWMA charts is of interest. Here, the effect of skewness of the underlying distribution on the performances of the Max-DEWMA and SS-DEWMA charts are compared. Various types of data ranging from nearly symmetric to highly skewed, representing a wide variety of shapes are generated. This study uses the Weibull, lognormal and gamma distributions to assess the robustness of the Max-DEWMA and SS-DEWMA control charts. A Monte Carlo simulation is performed herein to study the in-control Average Run Length $\left(\mathrm{ARL}_{0}\right)$ properties of the charts for various non-normal distributions.
The remainder of this study is organized as follows: first reviews the Max-DEWMA and SS-DEWMA control charts. Some statistical properties and design strategies are presented. A simulation study is conducted to compare the performances of the MaxDEWMA versus SS-DEWMA charts. Finally, concluding remarks are summarized .

The Max-DEWMA and SS-DEWMA control charts: Khoo et al. (2010) developed a single EWMA-type control chart, called the Max-DEWMA chart. Assume that a series of random observations, $X_{i j} \sim N\left(\mu+a \sigma, b^{2} \sigma^{2}\right)$, for $\mathrm{i}=1,2,3, \ldots$ and $\mathrm{j}=1,2,3$, $\ldots, n_{i}$, where $a$ and $b$ are constants and $b \geq 1$. The process is in-control when $a=0$ and $b=1$; otherwise the process has shifted. Let Eq. 1 and 2:

$\bar{X}_{i}=\frac{X_{i 1}+X_{i 2}+\ldots+X_{i n_{i}}}{n_{i}}$

and

$S_{i}^{2}=\frac{\sum_{j=1}^{n_{i}}\left(X_{i j}-\bar{X}_{i}\right)^{2}}{n_{i}-1}$

Be the mean and variance of sample $i$, respectively. The following two independent statistics are defined:

$U_{i}=\frac{\bar{X}_{i}-\mu}{\sigma / \sqrt{n_{i}}}$

$V_{i}=\Phi^{-1}\left\{H\left[\frac{\left(n_{i}-1\right) S_{i}^{2}}{\sigma^{2}} ; n_{i}-1\right]\right\}$

Note that in Eq. 3 and Eq. 4, $\Phi^{-1}(\cdot)$ denotes the inverse standard normal distribution function and $H(w$; $v$ ) the chi-square distribution function with $v$ degrees of freedom. When the process is in-control, both $U_{i}$ and $V_{i}$ in Eq. 3 and Eq. 4, respectively, are independent statistics having a common standard normal distribution. The two EWMA statistics computed from $U_{i}$ and $V_{i}$ are defined as follows:

$Y_{i}=(1-\lambda) Y_{i-1}+\lambda U_{i}$, for $i=1,2, \ldots$

and

$Z_{i}=(1-\lambda) Z_{i-1}+\lambda V_{i}$, for $i=1,2, \ldots$.

Here, $0<\lambda \leq 1$ is the smoothing constant, while $Y_{0}$ $=Z_{0}=0$ are the starting values of $Y_{i}$ and $Z_{i}$, respectively. 
From the two EWMA statistics of $Y_{i}$ and $Z_{i}$, given in Eq. 5-6, respectively, two corresponding DEWMA statistics can be computed as follows:

$$
W_{i}=(1-\lambda) W_{i-1}+\lambda Y_{i}, \text { for } i=1,2, \ldots
$$

And:

$$
Q_{i}=(1-\lambda) Q_{i-1}+\lambda Z_{i}, \text { for } i=1,2, \ldots
$$

Here, $W_{0}$ and $Q_{0}\left(W_{0}=Q_{0}=0\right)$ are the starting values of $W_{i}$ and $Q_{i}$, respectively. Khoo et al. (2010) uses the simple approach of setting the smoothing constants the same for $Y_{i}, Z_{i}, W_{i}$ and $Q_{i}$, in Eq. 5, Eq. 6, Eq. 7 and Eq. 8, respectively. Then the two DEWMA statistics in Eq. 7 and Eq. 8 are combined into the following single statistic for the proposed MaxDEWMA chart:

$M_{i}=\max \left\{\left|W_{i}\right|,\left|Q_{i}\right|\right\}$

Because the proposed chart is based on $M_{i}$, i.e., the maximum of $\left|W_{i}\right|$ and $\left|Q_{i}\right|$, it is called the Max-DEWMA chart. If the process mean has shifted away from its target value and/or the variance has increased or decreased, the statistic $M_{i}$, will be large. If both the process mean and variance stay close to their respective target values, then $M_{i}$ will be small. Note that $M_{i}$ is the maximum of the absolute values of the two DEWMA statistics and it is non-negative. Therefore, the MaxDEWMA chart needs only an Upper Control Limit (UCL ${ }_{\mathrm{MD}}$ ), which is given in Khoo et al. (2010).

$$
\begin{aligned}
& U C L_{M D}=E\left(M_{i}\right)+K_{M D} \sqrt{V\left(M_{i}\right)} \\
& =\left(1.128379+0.602810 K_{M D}\right) \sqrt{\frac{\lambda^{4}}{\left[1-(1-\lambda)^{2}\right]^{3}}} \\
& \times \sqrt{\left\{\begin{array}{l}
1+(1-\lambda)^{2}-\left(i^{2}+2 i+1\right) \\
(1-\lambda)^{2 i}+\left(2 i^{2}+2 i-1\right)(1-\lambda)^{2 i+2}-i^{2}(1-\lambda)^{2 i+4}
\end{array}\right\}}
\end{aligned}
$$

For $i=1,2, \ldots$, where $E\left(M_{i}\right)$ and $V\left(M_{i}\right)$ are the mean and variance of $M_{i}$, respectively, when the process is in-control, while $\mathrm{K}_{\mathrm{MD}}$ is a multiplier that controls the width of $\mathrm{UCL}_{\mathrm{MD}}$.

More recently, instead of taking the maximum of $\left|W_{i}\right|$ and $\left|Q_{i}\right|$, Teh et al. (2010a) also explored the idea of using the sum of squares of $W_{i}$ and $Q_{i}$ in Eq. 7 and
Eq. 8. They introduced the following single statistic for the proposed SS-DEWMA chart Eq. 9-11:

$$
L_{i}=W_{i}^{2}+Q_{i}^{2}
$$

Similar to the Max-DEWMA chart, the values of $L_{i}$ will be large if the mean and/or variance has shifted from their target values. However, $L_{i}$ will be small if both the mean and variance stay close to their respective target values.

Since $L_{i}$ is non-negative, the SS-DEWMA chart only has an upper control limit, given by:

$$
\begin{aligned}
& U C L_{S D}=E\left(L_{i}\right)+K_{S D} \sqrt{\operatorname{Var}\left(L_{i}\right)}, \\
& =2\left(1+K_{S D}\right) \times \frac{\lambda^{4}}{\left[1-(1-\lambda)^{2}\right]^{3}} \times \\
& \left\{\begin{array}{l}
1+(1-\lambda)^{2}-\left(i^{2}+2 i+1\right)(1-\lambda)^{2 i}+ \\
\left(2 i^{2}+2 i-1\right)(1-\lambda)^{2 i+2}-i^{2}(1-\lambda)^{2 i+4}
\end{array}\right\}
\end{aligned}
$$

For $i=1,2, \ldots$, where $E\left(L_{i}\right)$ and $\operatorname{Var}\left(L_{i}\right)$ are the incontrol mean and variance of $L_{i}$, respectively. Note that $K_{S D}$ is a constant controlling the width of $U C L_{S D}$. Eq. 12 enables a quick computation of $U C L_{S D}$ for the zero state SS-DEWMA chart, based on desired $\lambda$ and $K_{S D}$ values.

The derivation of $U C L_{S D}$ is shown in Teh et al. (2010b). As a rule of thumb, $\lambda$ in the interval $0.05 \leq \lambda \leq$ 0.30 is usually considered for a quick detection of small and moderate shifts in the mean and/or variance. Teh et al. (2010a) show that the SS-DEWMA chart performs better than the Max-DEWMA control chart in detecting shifts of all sizes in the mean and/or variance when the underlying distribution is normally distributed.

Statistical properties and design strategies: Skewness is a measure of the degree of asymmetry for a distribution. A distribution (or a data set) is symmetric if the median divides the left side and the right side into two identical regions. The sample skewness is measured with the following Eq. 13 (Kenney and Keeping, 1956):

Skewness $=\frac{\sum_{i=1}^{n}\left(X_{i}-\bar{X}\right)^{3}}{(n-1) S^{3}}$

where $n$ is the number of data points and $S$ the sample standard deviation. The skewness for a symmetric distribution has a value of zero. Negative values indicate data that are skewed to the left (left tail is longer relative to the right tail) and positive values 
indicate data that are skewed to the right (right tail is longer relative to the left tail).

The Weibull, lognormal and gamma distributions are considered in this study because these distributions are very flexible and by appropriate selection of the parameters, they can represent a wide variety of shapes, ranging from nearly symmetric to highly skewed. For convenience, a scale parameter of one is selected for both the Weibull and gamma distributions while a location parameter of zero is chosen for the lognormal distribution. This is because the skewness does not depend on the parameters of these distributions.

For a Weibull distribution, with a location parameter zero and scale parameter one, its cumulative distribution function (cdf) is given as Eq. 14:

$F(y)=1-e^{-(\omega y)^{\beta}}$, for $y \geq 0$

Where, $\omega>0$ is the scale parameter and $\beta>0$ the shape parameter. Note that when $\beta=1$, the Weibull distribution reduces to the exponential distribution with mean $\omega$. Letting $\omega=1$ and $P_{Y}=\operatorname{Pr}(Y \leq \mu)$, where $\mu$ is the target mean value of $Y$, we have:

$$
P_{Y}=1-\exp \left\{-\left[\Gamma\left(1+\frac{1}{\beta}\right)\right]^{\beta}\right\}, \text { for } y \geq 0
$$

For a lognormal distribution, its cdf is given as (Aitchison and Brown, 1969) Eq. 16:

$$
F(y)=\Phi\left(\frac{\log y-\theta}{\sigma_{L N}}\right), \text { for } y>0
$$

where $\theta$ is the location parameter and $\sigma_{\mathrm{LN}}$ the scale parameter. Letting $\theta=0$ and $P_{Y}=\operatorname{Pr}(Y \leq \mu)$, we have

$$
P_{Y}=\Phi\left(\frac{\sigma_{\mathrm{LN}}}{2}\right)
$$

The values of $\beta$ and $\sigma_{\mathrm{LN}}$ satisfying a given $\mathrm{P}_{\mathrm{Y}}$ in Eq. 15 and Eq. 17, respectively, can be obtained uniquely using a numerical method.

For a gamma distribution with a location parameter zero and scale parameter one, its cdf is given as (Johnson et al., 1994) Eq. 18:

$F(y)=\frac{\Gamma_{Y}(\alpha)}{\Gamma(\alpha)}$, for $y \geq 0, \alpha \geq 0$

where $\quad \Gamma_{Y}(\alpha)=\int_{0}^{y} m^{\alpha-1} e^{-m} d m$ and $\quad \Gamma(\alpha)=\int_{0}^{\infty} m^{\alpha-1} e^{-m} d m$. Then for this case Eq. 19:

$$
P_{Y}=F(\alpha)
$$

Since $\mu=\alpha$. Here, $\alpha$ denotes the shape parameter of the gamma distribution. Similar to the Weibull distribution, when $\alpha=1$, the gamma distribution reduces to the exponential distribution with mean 1 .

For the sake of comparison, besides the Weibull, lognormal and gamma distributions, the normal distribution is also considered. Note that the skewness coefficient, $\gamma$, is unique for a given value of $\beta, \sigma_{\mathrm{LN}}$ or $\alpha$. The shape parameters, $\beta$ for the Weibull distribution, $\sigma_{\mathrm{LN}}$ for the lognormal distribution and $\alpha$ for the gamma distribution, are determined so that the skewness coefficient, $\gamma=0.5(0.5) 3$. A skewness coefficient of zero indicates that the distribution is symmetry. The skewness coefficient, $\gamma=0.5$ and 1.0 represent low levels of skewness; $\gamma=1.5$ and 2.0 represent moderate levels of skewness; and $\gamma=2.5$ and 3.0 represent high levels of skewness. A shift in the mean is represented by $\mu_{Y, 1}=\mu_{Y, 0}+\delta \sigma_{Y, 0}$, where $\delta>0$ is the magnitude of a shift, in terms of the number of standard deviation units, while $\mu_{Y, 0}$ and $\sigma_{Y, 0}$ represent the in-control mean and in-control standard deviation, respectively. Note that we only consider the in-control process, i.e., when $\delta=0$. For a random variable, $Y$, from the Weibull, lognormal and gamma distributions, their in-control means are Eq. 20-22:

$$
\begin{aligned}
& \mu_{Y, 0}=\Gamma\left(1+\frac{1}{\beta}\right) \\
& \mu_{Y, 0}=e^{\frac{\sigma_{L N}^{2}}{2}}
\end{aligned}
$$

and

$$
\mu_{Y, 0}=\alpha
$$

Respectively, while their in-control standard deviations are Eq. 23-25:

$$
\begin{aligned}
& \sigma_{Y, 0}=\sqrt{\Gamma\left(1+\frac{2}{\beta}\right)-\left[\Gamma\left(1+\frac{1}{\beta}\right)\right]^{2}} \\
& \sigma_{Y, 0}=\sqrt{e^{\sigma_{L N}^{2}}\left(e^{\sigma_{L N}^{2}}-1\right)}
\end{aligned}
$$

and

$$
\sigma_{Y, 0}=\sqrt{\alpha}
$$


J. Math. \& Stat., 8 (1): 57-63, 2012

Table 1: In-control average run lengths for the Max-DEWMA and SS-DEWMA charts when $n=5$ and $\mathrm{ARL}_{0}=250$

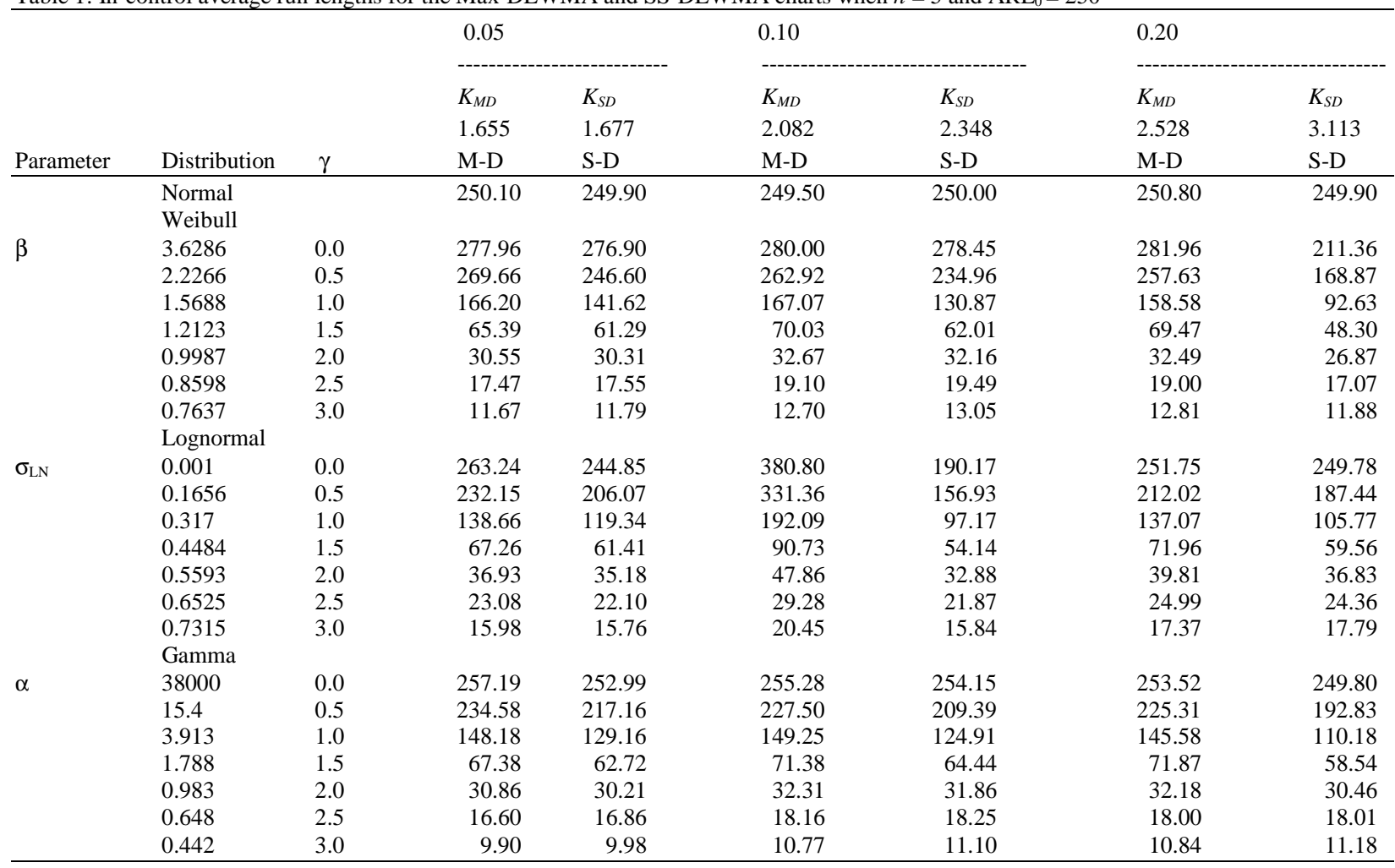

Table 1: Countinuous

\begin{tabular}{|c|c|c|c|c|c|c|c|c|c|}
\hline \multirow[b]{4}{*}{ Parameter } & \multirow[b]{4}{*}{ Distribution } & \multicolumn{2}{|l|}{0.30} & \multicolumn{2}{|l|}{0.50} & \multicolumn{2}{|l|}{0.80} & \multicolumn{2}{|l|}{1.00} \\
\hline & & $K_{M D}$ & $K_{S D}$ & $K_{M D}$ & $K_{S D}$ & $K_{M D}$ & $K_{S D}$ & $K_{M D}$ & $K_{S D}$ \\
\hline & & 2.799 & 3.585 & 3.090 & 4.188 & 3.246 & 4.492 & 3.250 & 4.528 \\
\hline & & M-D & S-D & M-D & S-D & M-D & S-D & M-D & S-D \\
\hline & $\begin{array}{l}\text { Normal } \\
\text { Weibull }\end{array}$ & 250.10 & 249.90 & 249.90 & 249.90 & 249.70 & 249.90 & 250.10 & 250.00 \\
\hline \multirow[t]{8}{*}{$\beta$} & 3.6286 & 296.24 & 291.55 & 305.55320 .12 & 339.29 & 344.62 & 346.49 & 354.03 & \\
\hline & 2.2266 & 263.68 & 203.77 & 255.63 & 191.28 & 237.78 & 168.50 & 225.11 & 164.91 \\
\hline & 1.5688 & 156.48 & 100.42 & 138.95 & 85.52 & 105.97 & 67.39 & 92.59 & 61.26 \\
\hline & 1.2123 & 69.43 & 50.82 & 63.96 & 43.30 & 51.07 & 34.61 & 44.97 & 32.16 \\
\hline & 0.9987 & 32.09 & 28.22 & 30.29 & 24.86 & 28.37 & 21.58 & 27.66 & 21.34 \\
\hline & 0.8598 & 18.74 & 17.88 & 17.69 & 16.03 & 17.54 & 14.94 & 18.79 & 15.81 \\
\hline & 0.7637 & 12.40 & 12.49 & 11.70 & 11.36 & 11.82 & 10.97 & 13.42 & 12.32 \\
\hline & Lognormal & & & & & & & & \\
\hline \multirow[t]{8}{*}{$\sigma_{\mathrm{LN}}$} & 0.001 & 255.21 & 249.95 & 251.08 & 250.55 & 252.67 & 249.78 & 250.81 & 249.92 \\
\hline & 0.1656 & 211.75 & 175.21 & 194.31 & 159.11 & 160.66 & 125.65 & 147.75 & 115.50 \\
\hline & 0.317 & 132.58 & 95.91 & 116.69 & 81.47 & 83.19 & 59.30 & 70.51 & 53.62 \\
\hline & 0.4484 & 72.13 & 55.65 & 67.95 & 49.21 & 50.78 & 37.23 & 44.10 & 33.85 \\
\hline & 0.5593 & 40.34 & 34.07 & 40.38 & 31.70 & 35.54 & 27.36 & 32.70 & 26.21 \\
\hline & 0.6525 & 24.91 & 23.09 & 25.73 & 22.64 & 26.53 & 21.50 & 26.67 & 21.93 \\
\hline & 0.7315 & 17.34 & 17.18 & 17.88 & 16.86 & 20.43 & 17.62 & 22.71 & 19.33 \\
\hline & Gamma & & & & & & & & \\
\hline \multirow[t]{7}{*}{$\alpha$} & 38000 & 258.45 & 250.78 & 253.41 & 254.86 & 253.69 & 254.92 & 249.89 & 257.14 \\
\hline & 15.4 & 220.82 & 180.38 & 202.76 & 161.12 & 169.00 & 131.42 & 153.30 & 119.90 \\
\hline & 3.913 & 143.46 & 98.30 & 125.55 & 82.53 & 90.31 & 61.19 & 77.90 & 55.17 \\
\hline & 1.788 & 70.96 & 52.36 & 65.06 & 44.67 & 50.92 & 35.50 & 45.20 & 32.81 \\
\hline & 0.983 & 31.76 & 27.95 & 30.04 & 24.29 & 27.60 & 21.06 & 27.31 & 21.03 \\
\hline & 0.648 & 17.44 & 16.81 & 16.25 & 15.06 & 15.61 & 13.54 & 16.61 & 14.16 \\
\hline & 0.442 & 10.53 & 10.74 & 9.69 & 9.65 & 9.10 & 8.76 & 9.91 & 9.44 \\
\hline
\end{tabular}


A comparison of the performances of the max-dewma versus ss-dewma charts: The effectiveness of a control chart in detecting a process change is measured by the Average Run Length (ARL), which is the expected number of samples plotted on a chart until the first outof-control signal is given (Montgomery, 2008). In other words, ARL is a measure of the speed of a control chart in detecting the occurrence of assignable causes (Zhang and Chen, 2005). The performances of the MaxDEWMA and SS-DEWMA charts are compared by designing charts with a common in-control ARL, ARL $=250$ and a sample size of $n=5$. As expected, other $\mathrm{ARL}_{0} \mathrm{~s}$ and sample sizes will also give similar results. A chart with an $\mathrm{ARL}_{0}$ value closer or equal to 250 for most of the cases is considered as the best chart. The normal, Weibull, lognormal and gamma distributions are considered in the computation of the $\mathrm{ARL}_{0} \mathrm{~S}$ of the Max-DEWMA and SS-EDWMA charts. The smoothing constant, $\lambda \in\{0.05,0.10,0.20,0.30,0.50,0.80,1.00\}$ is considered for both the Max-DEWMA and SSDEWMA charts. The various combinations of $\left(\lambda, K_{S D}\right)$ for the Max-DEWMA chart and $\left(\lambda, K_{S D}\right)$ for the SSDEWMA chart were obtained from Khoo et al. (2010) and Teh et al. (2010b), respectively. Here, the exact limits of both charts are considered.

Table 1 gives the $\mathrm{ARL}_{0} \mathrm{~S}$ for the Max-DEWMA and SS-DEWMA charts when $n=5$ and $\mathrm{ARL}_{0}=250$. Overall, the Max-DEWMA chart gives $\mathrm{ARL}_{0}$ values closer or equal to 250 compared to its SS-DEWMA counterpart in most of the cases, when the underlying population is skewed. For both the Max-DEWMA and SS-DEWMA control charts, when the level of positive skewness, $y$, increases, the probability of a sample point exceeding the control limit increases, hence the $\mathrm{ARL}_{0}$ decreases (or the Type I error rate increases).

This is true for all the three skewed distributions considered. For the normal population, the $\mathrm{ARL}_{0} \mathrm{~s}$ of both charts are close to 250. When the skewness level, $\gamma$ equals to zero, the distribution of the data is symmetric, therefore, the $\mathrm{ARL}_{0} \mathrm{~s}$ for the Weibull, lognormal and gamma distributions approach the $\mathrm{ARL}_{0}$ value of a normal distribution, i.e., 250.

\section{CONCLUSION}

Concluding remarks: In most cases of skewed populations, irrespective of the skewness, $\gamma$, smoothing constant, $\lambda$ and type of skewed distribution, the MaxDEWMA chart produces $\mathrm{ARL}_{0}$ values that are closer or equal to 250 than the SS-DEWMA chart.

Although Teh et al. (2010a) showed that the SSDEWMA chart outperforms the Max-DEWMA chart, in terms of the out-of-control detection speed and diagnostic abilities, when the underlying distribution is normal; this conclusion is no longer true when the underlying distribution is skewed. This is indicated by the results in the "A Comparison Of The Performances Of The Max-DEWMA Versus SS-DEWMA Charts" section. Therefore, the SS-DEWMA chart can act as a favorable substitute to the existing single EWMA charts for a joint monitoring of the mean and/or variance only if the distribution of the data is normally distributed. Practitioners are advised to employ the Max-DEWMA chart for a joint monitoring of the mean and/or variance, when the underlying distribution is non-normal:

- Among the potential future works on this topic that are worth pursuing are as follows

- To compare the performances of the SS-DEWMA and Max-DEWMA charts for skewed populations, in terms of the Median Run Length (MRL), Standard Deviation of the Run Length (SDRL) and percentage points of the run length distribution

- To investigate the performances of the SSDEWMA and Max-DEWMA charts for the steady state mode process, as we only consider the zero state mode process in this study

- To measure the performances of the SS-DEWMA and Max-DEWMA charts when their smoothing constants, $\lambda$ 's, have different weights

- To study the performances of the SS-DEWMA and Max-DEWMA charts, based on an autocorrelated process, i.e., when the independence assumption is violated

- To evaluate the performances of the SS-DEWMA and Max-DEWMA charts, based on heavy tailed distributions, such as the Student- $t$ or Cauchy distributions

\section{ACKNOWLEDGMENT}

This stydy is carried out in the School of Mathematical Sciences, Universiti Sains Malaysia (USM) and is supported by the Academic Staff Training Scheme (ASTS), School of Management, USM and the USM-RU-PRGS fund.

\section{REFERENCES}

Aitchison, J. and J.A.C. Brown, 1969. The Lognormal Distribution: With Special Reference to its Uses in Economics. 1st Edn., University Press, Cambridge, pp: 176.

Amhemad, A.Z., 2009. Effect of non normality on statistical control charts. Proceedings of the International Conference on Networking and Information Technology, Jun. 11-12, IEEE Explore Press, Manila, pp: 512-515. DOI: 10.1109/ICNIT.2010.5508459 
Besterfield, D.H. 2009. Quality Control. 8th Edn. Pearson/Education Hall, Upper Saddle River, New Jersey, ISBN: 0135000955, pp: 540.

Chen, G., S.W. Cheng and H. Xie, 2001. Monitoring process mean and variability with one EWMA Chart. J. Qual. Technol., 33: 223-233.

Duncan, A.J., 1986. Quality Control and Industrial Statistics. 5th. Edn., Irwin, Homewood, ILL, ISBN10: 0256035350 , pp: 1123.

Gupta, B.C. and H.F. Walker, 2007. Statistical Quality Control for the Six Sigma Green Belt. ASQ Quality Press, Milwaukee, Wisconsin, ISBN: 9780873896863, pp: 340.

Hai-yu, W. and X. Ji-chao, 2007. Statistical Process Control for Small Shifts Based on Skewed Distribution. Proceedings of the 14th International Conference on Management Science and Engineering, Aug 20-22, IEEE Xplore Press, Harbin, pp: 543-548. DOI: 10.1109/ICMSE.2007.4421903

Johnson, N.L., S. Kotz and N. Balakrishnan, 1994. Continuous Univariate Distributions. 2nd Edn., Wiley and Sons, New York, ISBN-10: 0471584959, pp: 784.

Kenney, J.F. and E.S. Keeping, 1956. Mathematics of Statistics. 2nd Edn., Van Nostrand, New York, pp: 429.

Khoo, M.B.C. and M. Kassim, 2008. A Comparison of Performances of Three Types of Control Charts for Skewed Distributions. Matematika, 24: 169-185.

Khoo, M.B.C., S.Y. Teh and Z. Wu, 2010. Monitoring Process mean and variability with one double EWMA chart. Commun. Stat.-Theor. Meth., 39: 3678-3694. DOI: 10.1080/03610920903324866
Khoo, M.B.C., Z. Wu and A.M.A. Atta, 2008. A synthetic control chart for monitoring the process mean of skewed populations based on the weighted variance method. Int. J. Reliab. Qual. Saf. Eng., 15: 217-245. DOI: 10.1142/S0218539308003052

Montgomery, D.C., 2008. Introduction to Statistical Quality Control. 6th Edn., Wiley, Hoboken, ISBN: 0470169923, pp; 734.

Oakland, J.S., 2003. Statistical Process Control. 5th Edn., Butterworth-Heinemann, Burlington, MA., ISBN-10: 0750657669, pp: 445.

Roberts, S.W., 1959. Control chart tests based on geometric moving averages. Technometrics, 1: 239-250.

Teh, S.Y., M.B.C. Khoo and L.C. Kit, 2010a. Comparing the performances of the optimal SSDEWMA and max-DEWMA Control. Chart. J. Stat. Modl. Anal., 1: 1-9.

Teh, S.Y., M.B.C. Khoo and Z. Wu, 2010b. A sum of squares double exponentially weighted moving average chart. Comput. Ind. Eng., 61: 1173-1188.

Umble, E.J. and M.M. Umble, 2000. Developing control charts and illustrating type $\mathrm{i}$ and type ii errors. Qual. Manage. J., 7: 23-30.

Xie, H., 1999. Contributions to Qualimetry. University of Manitoba Libraries.

Zhang, L. and G. Chen, 2005. An extended EWMA mean chart. Qual. Technol. Quantit. Manage., 2: 39-52. 